ESAIM: PROCEEDINGS, July 2007, Vol.18, 216-228

Jean-Frédéric Gerbeau \& Stéphane Labbé, Editors

\title{
TRANSFORMÉE EN ONDELETTES CONTINUE DIRECTIONNELLE : APPLICATIONS EN IMAGERIE MÉDICALE*
}

\author{
Valérie Perrier ${ }^{1}$, Anne Bilgot ${ }^{2}$, Olivier Le Cadet $^{1}$ et Laurent Desbat ${ }^{2}$
}

\begin{abstract}
Résumé. Cet article est issu d'un mini-cours donné à l'école doctorale de l'université d'Orsay en décembre 2005. Nous présentons la transformée en ondelettes continue directionnelle en dimension 2, et la formule de synthèse générale associée. Nous insistons sur deux exemples fondamentaux d'ondelettes en traitement d'image, et proposons un calcul pratique des coefficients. Nous illustrons ensuite cette théorie par deux applications nouvelles en imagerie médicale, liées à des problèmes de reconstruction d'objets à partir de données incomplètes : la tomographie locale, et l'identification d'une surface de vertèbre à partir de deux radiographies.
\end{abstract}

\begin{abstract}
This article comes from a short lecture given at the Graduate School of Paris Orsay's university during December, 2005. We present the directional continuous wavelet transform in dimension 2, and the general associated synthesis formula. We develop two fundamental examples of wavelets in image processing, and propose a practical method for the coefficients calculation. We illustrate this theory by two original applications in medical imaging, related to reconstruction of objects from incomplete data: local tomography and the identification of a vertebra surface from two X-ray images.
\end{abstract}

\section{INTRODUCTION}

L'imagerie médicale a révolutionné les pratiques médicales. Néanmoins, de nombreux problèmes liés au traitement d'image y sont encore ouverts et leur résolution (même partielle) peut aboutir à une amélioration des diagnostics et actes chirurgicaux. On peut citer par exemple : le problème de la réduction des radiations administrées lors d'un examen scanner (problème de la tomographie locale), la chirurgie assistée par ordinateur (incluant des problèmes de segmentation automatique, de recalage de données et de reconstruction temps-réel 3D), ou encore la détection et l'analyse de structures malignes dans des données d'échographie, mammographie, ou spectroscopie RMN (incluant par exemple l'analyse d'images texturées), etc.

L'utilisation des bases d'ondelettes en traitement d'images s'est généralisée durant les vingt dernières années [3]. Leur intérêt pour la compression et le débruitage a été démontré puisqu'elles ont intégré le dernier standard de compression des images numériques JPEG 2000. Leur application à l'imagerie médicale date de 1992 et s'est largement répandue depuis $[1,2]$. Dans ce contexte les ondelettes sont utilisées pour la compression et le débruitage, mais aussi pour l'analyse fonctionnelle de données médicales (en vue d'établir un diagnostic), la tomographie locale, la segmentation et le rehaussement d'images, ou encore la description de textures. Nous nous intéressons ici à la contribution des ondelettes pour la reconstruction (locale et globale) de structures anatomiques à partir de projections : pour la tomographie locale et la segmentation automatique de radiographies en chirurgie interventionnelle.

Pour les applications classiques que sont la compression d'images (JPEG2000), le débruitage, ou encore des méthodes de réduction de dimension en vue de la classification [7], l'outil utilisé est la décomposition sur bases d'ondelettes d'une fonction (bases orthonormées ou bases biorthogonales). Pour l'analyse d'images et la caractérisation de structures dans l'image (contours, textures) une transformée redondante apporte l'avantage d'une information plus facile à analyser et à interpréter. Les applications que nous allons développer dans la suite de cet article concernent la transformée en ondelettes continue bidimensionnelle, ou sa version discrétisée en échelle : la transformée dyadique.

* Ces travaux ont été soutenus par la CEE (projets IST-1999-12338 MI3 et HPRN-CT-2002-00286 Breaking Complexity) ainsi que par la Région Rhône-Alpes

${ }^{1}$ Laboratoire Jean Kuntzmann, Institut National Polytechnique de Grenoble, BP 53, 38041 Grenoble Cedex 9

${ }^{2}$ Laboratoire de Techniques de l'Imagerie, de la Modélisation et de la Cognition de l'IMAG, Institut de l'Ingénierie de

l’Information de Santé, Faculté de Médecine, Domaine de la Merci, 38706 La Tronche 
Au chapitre 2 nous rappelons les ingrédients principaux de la transformée en ondelettes bidimensionnelle. Les chapitres suivants concernent deux applications en imagerie médicale : le problème de la reconstruction locale (section 3) et celui de la segmentation automatique de radiographie en vue de la reconstruction $3 \mathrm{D}$ (section4).

\section{TRAnSFORMÉE En Ondelettes CONTINUE DiRECTIONNELlE}

La transformée en ondelette continue directionnelle, en dimension 2, a été originellement introduite par Murenzi [25]. Les propriétés et applications de la transformée en ondelettes continue sont détaillées dans $[4,6]$. A une fonction donnée, cette transformation fait correspondre un coefficient dépendant d'une position $\mathbf{b}$, d'une échelle a, et d'une orientation $\alpha$. Nous supposerons dans la suite que toutes les ondelettes sont des fonctions réelles.

La décomposition en ondelettes d'une fonction $f \in L^{2}\left(\mathbb{R}^{2}\right)$ contre une ondelette d'analyse $g \in$ $L^{2}\left(\mathbb{R}^{2}\right)$ est définie pour tout $a>0, \mathbf{b} \in \mathbb{R}^{2}, \alpha \in[0,2 \pi]$, par

$$
W_{g} f(a, \mathbf{b}, \alpha)=\iint_{\mathbb{R}^{2}} f(\mathbf{x}) \frac{1}{a} g\left(r_{-\alpha}\left(\frac{\mathbf{x}-\mathbf{b}}{a}\right)\right) \mathbf{d x}
$$

où $r_{-\alpha}$ désigne la rotation d'angle $(-\alpha)$ dans $\mathbb{R}^{2}$.

Il est alors possible de reconstruire $f$ à partir de ses coefficients d'ondelettes $W_{g} f(a, \mathbf{b}, \alpha)$, en utilisant une ondelette de synthèse $h$, c'est-à-dire une fonction de $L^{2}\left(\mathbb{R}^{2}\right)$ vérifiant la condition d'admissibilité ${ }^{1}$ :

$$
C_{g h}=\iint_{\mathbb{R}^{2}} \frac{\overline{\hat{g}}(\mathbf{k}) \hat{h}(\mathbf{k})}{\|\mathbf{k}\|^{2}} \mathbf{d} \mathbf{k}<+\infty
$$

On obtient alors la formule de synthèse contre l'ondelette de reconstruction $h$ :

$$
f(\mathbf{x})=\frac{1}{C_{g h}} \int_{0}^{+\infty} \iint_{\mathbb{R}^{2}} \int_{0}^{2 \pi} W_{g} f(a, \mathbf{b}, \alpha) \frac{1}{a} h\left(r_{-\alpha}\left(\frac{\mathbf{x}-\mathbf{b}}{a}\right)\right) \frac{d a}{a^{3}} \mathbf{d} \mathbf{b} d \alpha
$$

Dans le cas où l'ondelette d'analyse est auto-admissible, i.e. vérifie :

$C_{g g}=\iint_{\mathbb{R}^{2}} \frac{|\hat{g}(\mathbf{k})|^{2}}{\|\mathbf{k}\|^{2}} \mathbf{d k}<+\infty$, on a également la conservation de l'énergie :

$$
\iint_{\mathbb{R}^{2}}|f(\mathbf{x})|^{2} \mathbf{d x}=\frac{1}{C_{g g}} \int_{0}^{+\infty} \iint_{\mathbb{R}^{2}} \int_{0}^{2 \pi}\left|W_{g} f(a, \mathbf{b}, \alpha)\right|^{2} \frac{d a}{a^{3}} \mathbf{d} \mathbf{b} d \alpha
$$

ce qui signifie que la transformée en ondelettes continue est une isométrie de $L^{2}\left(\mathbb{R}^{2}\right)$ dans $L^{2}\left(\mathbb{R}^{+} \times \mathbb{R}^{2} \times[0,2 \pi], \frac{d a}{a^{3}} \mathbf{d b} d \alpha\right)$.

On remarquera qu'une fonction $g$, à la fois intégrable et de carré intégrable, est une ondelette autoadmissible si et seulement si elle est de moyenne nulle : $\iint_{\mathbb{R}^{2}} g(\mathbf{x}) \mathbf{d} \mathbf{x}=0$. Dans la pratique, une catégorie d'ondelettes couramment utilisée est celle des dérivées de Gaussienne. En particulier, les deux exemples suivants sont fondamentaux en traitement d'images.

Exemple 1 : le Laplacien de Gaussienne $\Delta \mathrm{G}$. Le Laplacien de Gaussienne est un filtre couramment utilisé en traitement d'images ou en vision par ordinateur. C'est un détecteur de bord classique, pour lequel la recherche des bords (ou contours) dans une image se fait en analysant les passages par zéro de l'image convoluée par ce filtre.

Pour la transformée en ondelettes on utilise souvent $g=h=\Delta G$ où $G(x, y)=e^{-\pi\left(x^{2}+y^{2}\right)}$, ce qui revient à faire la convolution de l'image $f$ par un Laplacien de Gaussienne à différentes échelles $a$. Dans ce cas l'ondelette est isotrope et la dépendance en $\alpha$ disparat dans la définition de la décomposition (1), et la formule de synthèse (2), qui devient :

$$
f(\mathbf{x})=\frac{2}{\pi} \int_{0}^{+\infty} \iint_{\mathbb{R}^{2}} W_{\Delta G} f(a, \mathbf{b}) \frac{1}{a} \Delta G\left(\frac{\mathbf{x}-\mathbf{b}}{a}\right) \frac{d a}{a^{3}} \mathbf{d} \mathbf{b}
$$

Exemple 2 : le Gradient de Gaussienne $\nabla$ G. Le gradient de Gaussienne est un détecteur de bord classique, car convoluer une image par $\nabla \mathbf{G}$ revient à analyser le gradient de l'intensité lissée par une Gaussienne. Dans [12], Canny introduit un détecteur encore beaucoup utilisé : après convolution de l'image par une Gaussienne, on calcule son gradient pour rechercher les points correspondant aux fortes variations de l'intensité, repérés comme les maxima locaux de ce gradient, dans la direction du gradient.

$1_{\text {ici }} \hat{g}$ désigne la transformée de Fourier de la fonction $g$ définie par $\hat{g}(\mathbf{k})=\iint_{\mathbb{R}^{2}} g(\mathbf{x}) e^{-2 i \pi \mathbf{k} \cdot \mathbf{x}} \mathbf{d k}$ 
L'utilisation du gradient de Gaussienne comme ondelette pour caractériser des contours multi-échelles a été introduite par Mallat [24]. La transformée en ondelettes d'une fonction $f$ avec une ondelette d'analyse $\mathbf{g}=\nabla \mathbf{G}$ est alors vectorielle et définie pour tout $a>0, \mathbf{b} \in \mathbb{R}^{2}$ par

$$
\mathbf{W}_{\nabla \mathbf{G}} f(a, \mathbf{b})=\iint_{\mathbb{R}^{2}} f(\mathbf{x}) \frac{1}{a} \nabla \mathbf{G}\left(\frac{\mathbf{x}-\mathbf{b}}{a}\right) \mathbf{d x}
$$

Cette transformée est reliée à la transformée directionnelle (1) si l'on choisit comme ondelette d'analyse $g=\frac{\partial G}{\partial x}$. En effet, on a la relation

$$
W_{\frac{\partial G}{\partial x}} f(a, \mathbf{b}, \alpha)=\mathbf{u}_{\alpha} \cdot \mathbf{W}_{\{\nabla \mathbf{G}\}} f(a, \mathbf{b})
$$

où $\mathbf{u}_{\alpha}$ désigne le vecteur unitaire dans la direction $\alpha: \mathbf{u}_{\alpha}=(\cos \alpha, \sin \alpha)$, et "." le produit scalaire euclidien dans $\mathbb{R}^{2}$. La formule de synthèse associée à la transformée en ondelettes vectorielle (5) se déduit alors de (2), en prenant $h=g=\frac{\partial G}{\partial x}[20]$ :

$$
f(\mathbf{x})=\frac{1}{\pi} \int_{0}^{+\infty} \iint_{\mathbb{R}^{2}}\left[\mathbf{W}_{\nabla \mathbf{G}} f(a, \mathbf{b}) \cdot \frac{1}{a} \nabla \mathbf{G}\left(\frac{\mathbf{x}-\mathbf{b}}{a}\right)\right] \frac{d a}{a^{3}} \mathbf{d b}
$$

De manière générale, on peut définir des ondelettes directionnelles en s'inspirant de filtres sélectifs utilisés en traitement d'images, comme par exemple les "steerable filters" introduits par Freeman et Adelson [18].

\subsection{Transformée en ondelettes dyadique}

Le problème se pose de la discrétisation des formules de décompositions précédentes, en vue du calcul pratique des coefficients d'ondelettes d'une fonction. Dans le cas particulier d'une discrétisation en échelle dyadique, $a=2^{j}, j \in \mathbb{Z}$, on montre que moyennant une hypothèse supplémentaire sur l'ondelette de synthèse, on obtient des formules de reconstructions exactes. C'est la transformée en ondelettes dyadique, définie par S. Mallat [3].

La transformée en ondelettes directionnelle dyadique d'une fonction $f \in L^{2}\left(\mathbb{R}^{2}\right)$ contre une ondelette d'analyse (réelle) $g \in L^{2}\left(\mathbb{R}^{2}\right)$ est définie pour tout $j \in \mathbb{Z}, \mathbf{b} \in \mathbb{R}^{2}, \alpha \in[0,2 \pi]$, par

$$
W_{g} f\left(2^{j}, \mathbf{b}, \alpha\right)=\iint_{\mathbb{R}^{2}} f(\mathbf{x}) 2^{-j} g\left(2^{-j} r_{-\alpha}(\mathbf{x}-\mathbf{b})\right) \mathbf{d} \mathbf{x}
$$

Une ondelette de synthèse pour cette transformée est une fonction $h$ vérifiant :

$$
\int_{0}^{2 \pi}\left[\sum_{j \in \mathbb{Z}} \overline{\hat{g}\left(2^{j} r_{-\alpha} \mathbf{k}\right)} \hat{h}\left(2^{j} r_{-\alpha} \mathbf{k}\right)\right] d \alpha=1, \quad \forall \mathbf{k} \in \mathbb{R}^{2}-\{\mathbf{0}\}
$$

On a alors la formule de reconstruction :

$$
f(\mathbf{x})=\sum_{j=-\infty}^{+\infty} 4^{-j} \iint_{\mathbb{R}^{2}} \int_{0}^{2 \pi} W_{g} f\left(2^{j}, \mathbf{b}, \alpha\right) 2^{-j} h\left(2^{-j} r_{-\alpha}(\mathbf{x}-\mathbf{b})\right) \mathbf{d} \mathbf{b} d \alpha
$$

Exemple 1 : le Laplacien de Gaussienne $\Delta \mathrm{G}$. Pour une ondelette $g=\Delta G$, la formule de synthèse (7) se simplifie :

$$
f(\mathbf{x})=\frac{1}{2 \pi} \sum_{j=-\infty}^{+\infty} 4^{-j} \iint_{\mathbb{R}^{2}} W_{\Delta G} f\left(2^{j}, \mathbf{b}\right) 2^{-j} h\left(2^{-j}(\mathbf{x}-\mathbf{b})\right) \mathbf{d b}
$$

en prenant par exemple

$$
\hat{h}(\mathbf{k})=\frac{e^{-\pi\|\mathbf{k}\|^{2}}}{4 \pi^{2} \sum_{j=-\infty}^{+\infty} 4^{j}\|\mathbf{k}\|^{2} e^{-2 \pi 4^{j}\|\mathbf{k}\|^{2}}}
$$

Exemple 2 : le Gradient de Gaussienne $\nabla \mathbf{G}$. Dans le cas d'une ondelette $\mathbf{g}=\nabla \mathbf{G}$, la formule de synthèse s'écrit :

$$
f(\mathbf{x})=\sum_{j=-\infty}^{+\infty} 4^{-j} \iint_{\mathbb{R}^{2}}\left[\mathbf{W}_{\nabla \mathbf{G}} f\left(2^{j}, \mathbf{b}\right) \cdot 2^{-j} \mathbf{h}\left(2^{-j}(\mathbf{x}-\mathbf{b})\right)\right] \mathbf{d b}
$$


avec une ondelette de synthèse $\mathbf{h}$ vectorielle, définie par sa transformée de Fourier en coordonnées polaires :

$$
\hat{\mathbf{h}}(\rho \cos \theta, \rho \sin \theta)=\varphi(\rho) \mathbf{u}_{\theta}
$$

où la fonction $\varphi$ est définie par :

$$
\varphi(\rho)=\frac{i \rho e^{-\pi \rho^{2}}}{2 \pi \sum_{j=-\infty}^{+\infty} 4^{j} \rho^{2} e^{-2 \pi 4^{j} \rho^{2}}}
$$

On remarquera que la fonction $F(\rho)=\sum_{j=-\infty}^{+\infty} 4^{j} \rho^{2} e^{-2 \pi 4^{j} \rho^{2}}$ intervenant aux dénominateurs des ondelettes de synthèses choisies dans les 2 exemples, est toujours strictement positive en dehors de $\rho=0$, et faiblement oscillante autour de la valeur $1 /(4 \pi \ln 2)$ (et peut être considérée comme constante en première approximation).

\subsection{Algorithmes rapides}

Dans les algorithmes, l'image $f$ est discrétisée sur $2^{J} \times 2^{J}$ valeurs entières, et sa transformée en ondelettes dyadique est calculée pour les échelles $2^{j}, j=0,2, \cdots J-1$, sans décimation en espace. Pour les ondelettes d'analyse issues des exemples 1 et 2 , les coefficients à l'échelle $2^{j}$ sont calculés par des convolutions discrètes successives de $f$ avec un filtre passe-bas $h$ (remplaçant la convolution continue avec la Gaussienne G), suivies de convolutions avec des filtres discrets de dérivation $g_{x}$ (dérivée suivant $x$ ) ou $g_{y}$ (dérivée suivant y). Par exemple :

$$
h=\frac{1}{16}\left(\begin{array}{ccc}
1 & 2 & 1 \\
2 & 4 & 2 \\
1 & 2 & 1
\end{array}\right), \quad g_{x}=\frac{1}{2}[0,1,-1], \quad g_{y}=\frac{1}{2}\left[\begin{array}{c}
0 \\
1 \\
-1
\end{array}\right] \text {. }
$$

Dans le cas de l'exemple 2 , les coefficients $\mathbf{W}_{\{\nabla \mathbf{G}\}} f\left(2^{j},(\mathbf{n}, \mathbf{m})\right)$ sont calculés en pratique par :

$$
\mathbf{W}_{\{\nabla \mathbf{G}\}} f=\left(W^{1} f, W^{2} f\right) \text { où } W^{1} f=f * h * \cdots * h * g_{x} \text { et } W^{2} f=f * h * \cdots * h * g_{y}
$$

Le nombre de convolutions du filtre $h$ est lié à la propriété de la Gaussienne :

$G * \cdots * G(n$ facteurs $)=\frac{1}{n} G\left(\frac{x}{\sqrt{n}}, \frac{y}{\sqrt{n}}\right)$. Pour calculer une échelle dyadique $\sqrt{n}=2^{j}$, on effectue $2^{2 j}-1$ convolutions du filtre $h$. Le calcul complet des coefficients d'ondelettes à toutes les échelles de la forme $\sqrt{n}$ (avec $n$ entier) a une complexité de $O\left(2^{2 J}\right)$ opérations. On peut associer à cette décomposition approchée, une formule de synthèse discrète, pour ce choix particulier de filtres discrets, qui fait directement intervenir des filtres splines biorthogonaux [20,24].

\section{Ondelettes et Tomographie locale}

L'avènement du scanner a permis de fournir des informations pré-opératoires précises sur le patient qui servent à la fois à établir un diagnostic, à élaborer un planning chirurgical mais aussi à guider le chirurgien. Toutefois l'examen scanner, s'il est efficace pour la reconstruction 3D de structures anatomiques, est un examen irradiant. L'objectif médical est d'une part de réduire au minimum les radiations administrées au patient; d'autre part, en condition opératoire, les détecteurs des appareils de mesure ont des tailles réduites et ne permettent de mesurer que des données tronquées des structures. Dans ce qui suit, nous nous placerons en géométrie parallèle.

\subsection{Principe de fonctionnement d'un scanner}

Le principe de fonctionnement d'un scanner est celui de l'acquisition de données radiographiques sur des détecteurs (appelées également projections tomographiques), positionnés autour de l'objet à étudier (figure 1). Les géométries d'acquisition peuvent être différentes suivant les types d'appareils : acquisition circulaire à géométrie parallèle (les faisceaux de rayons X arrivant sur les détecteurs sont parallèles) à géométrie conique, ou encore à acquisition hélicodale.

En dimension 2 d'espace, ces données radiographiques correspondent mathématiquement à la transformée de Radon de la fonction d'atténuation, mesurée sur chaque détecteur.

La transformée de Radon de la fonction d'atténuation $f(\mathbf{x})$ sur un détecteur correspond à la moyenne de $f$ le long des droites $L_{\theta, s}$ (cf figure 1 , droite) : pour $s \in \mathbb{R}$,

$$
\mathcal{R}_{\theta} f(s)=\int_{L_{\theta, s}} f(\mathbf{x}) d \ell=\int_{-\infty}^{+\infty} f\left(s \mathbf{u}_{\theta}+t \mathbf{u}_{\theta}^{\perp}\right) d t
$$



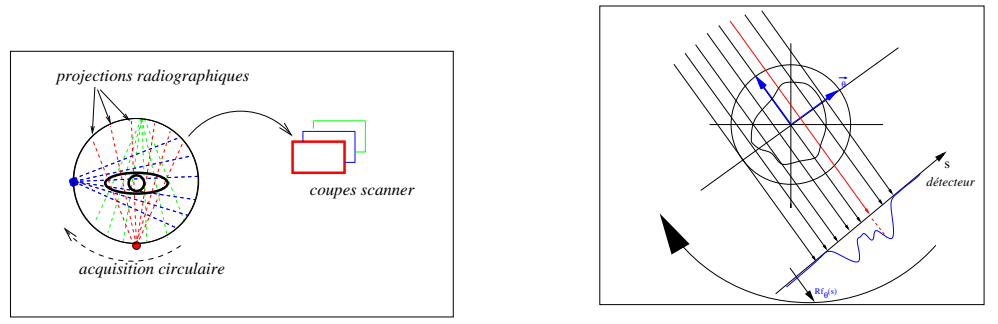

FIGURE 1. Le principe de fonctionnement d'un scanner : acquisition circulaire de données radiographiques autour de la section d'intérêt (à gauche), et principe de mesure de la transformée de Radon sur un détecteur (à droite)

où $\mathbf{u}_{\theta}=(\cos \theta, \sin \theta)$ et $\mathbf{u}_{\theta}^{\perp}=(-\sin \theta, \cos \theta)$.

Pour reconstruire une structure anatomique en dimension 2 à partir de ses projections, on doit inverser la transformée de Radon. La formule d'inversion classique, et utilisée en pratique, fait appel à la transformée de Fourier, c'est la formule de rétro-projection filtrée [5] :

$$
f(\mathbf{x})=\int_{0}^{\pi} \int_{-\infty}^{+\infty} \widehat{\mathcal{R}_{\theta} f}(\omega)|\omega| e^{2 i \pi \mathbf{x} \cdot \mathbf{u}_{\theta}} d \theta d \omega, \quad \forall \mathbf{x} \in \mathbb{R}^{2}
$$

(ici $\hat{g}$ désigne la transformée de Fourier d'une fonction unidimensionnelle $g$ ).

L'inconvénient de cette formule est que la reconstruction de la fonction en un point nécessite la connaissance de la transformée de Radon sur tous les détecteurs. L'objectif consiste alors à développer de nouvelles méthodes de reconstructions moins invasives, en cherchant des formules de reconstructions locales pour remplacer (9).

\subsection{Tomographie locale}

Le problème se pose de la reconstruction d'une structure $2 \mathrm{D}$, à partir de données tronquées de sa transformée de Radon. Nous considérerons ici le problème intérieur : comment reconstruire une fonction $f(\mathbf{x})$ en tout point du disque $\|\mathbf{x}\|<a$ (appelé Région d'Intérêt ou ROI), à partir de ses projections $\mathcal{R}_{\theta} f(s)$ pour $|s|<a$ et $\theta \in[0,2 \pi]$ ? (ce qui correspond à reconstruire $f$ dans la région d'intérêt en concentrant les rayons dans cette région, comme c'est schématisé à la figure 2(b)).

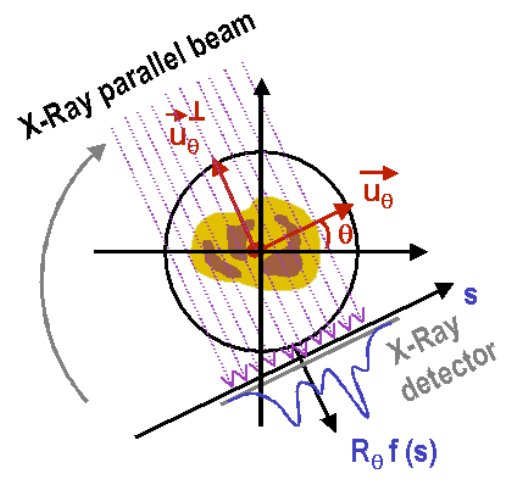

(a) Acquisition et paramétrisation de la Transformée de Radon

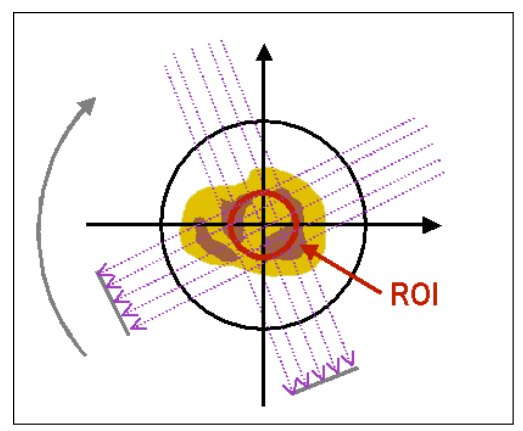

(b) Le problème intérieur : seules les mesures de la Transformée de Radon traversant la région d'intérêt sont prises en compte.

FiguRE 2. La Transformée de Radon pour des données globales et tronquées.

En dimension 2 d'espace, le problème intérieur est mathématiquement mal posé, car il n'y a pas unicité de la solution (en effet deux fonctions peuvent avoir la même transformée de Radon à travers la région d'intérêt, sans être égales sur cette région). Toutefois, on peut montrer que deux solutions diffèrent à peu près d'une constante, dans un disque un peu plus petit que la région d'intérêt [5]. La solution est alors de considérer les projections d'une région un peu plus grande que la région d'intérêt, appelée région d'exposition ou ROE (dans ce cas on considère $\|\mathbf{x}\|<a+\delta a$ ), afin de reconstruire à une constante près $f$, dans la région d'intérêt. On se tourne alors vers des formulations locales de reconstruction. 


\subsection{Inversion de la Transformée de Radon par Ondelettes}

Une réponse au problème de l'inversion locale est apportée par les formules d'inversion de la transformée de Radon dans des bases d'ondelettes, et l'introduction des ondelettes en tomographie date du début des années 90 : par analogie avec la méthode de Fourier, on peut calculer la transformée en ondelettes 2D d'une fonction par "rétroprojection" de la transformée en ondelettes (1D) de sa transformée de Radon sur chaque détecteur; l'ondelette génératrice 1D associée aux détecteurs et l'ondelette génératrice 2D sont liées par une relation faisant intervenir la transformée de Radon $[8,27]$. Cette première approche a été développée dans le but de faire de la tomographie locale, et nous proposons une comparaison pour le problème intérieur à la figure 5 .

L'approche par ondelettes/vaguelettes définie par D. Donoho [15] a pour objectif d'obtenir des reconstructions stables, sur bases d'ondelettes 2D, pour des données bruitées. Le principe de cette approche s'apparente à la SVD (Singular Value Decomposition), en remplaçant les vecteurs propres par une base d'ondelettes 2D indépendante de l'opérateur : les coefficients sur cette base orthogonale d'ondelettes 2D de la fonction sont alors fournis par les coefficients de sa transformée de Radon sur base de "vaguelettes" 2D biorthogonales. De la même manière que dans l'approche précédente, les vaguelettes sont reliées aux ondelettes par la transformée de Radon. Bien que pouvant s'appliquer en théorie au problème de la tomographie locale, cette méthode a été utilisée pour des reconstructions globales (voir par exemple [21]).

L'approche par ondelettes/vaguelettes a été ensuite étendue aux nouvelles bases d'ondelettes "géométriques" (ridgelets, curvelets [13], bandelettes [22, 26]), développées dans un premier temps pour la compression d'images. L'intérêt de ces nouvelles bases, outre celui de fournir des reconstructions stables, est de pouvoir préserver les contours de la fonction après le débruitage (en pratique un seuillage sur les coefficients). Ces approches apparaissent donc comme très attractives dans le contexte de l'imagerie médicale où les fonctions à reconstruire sont des fonctions constantes par morceaux, comme le célèbre fantôme de SheppLogan (figure 4) : ici l'information importante réside dans les discontinuités. Ces approches par ridgelets, curvelets et bandelettes ont été appliquées avec succès pour la reconstruction globale d'images (fantômes) bruitées, et les discontinuités sont mieux reconstruites qu'avec des ondelettes tensorielles [13,14,26]. Malheureusement les algorithmes rapides actuellement accessibles pour ces méthodes passent par le domaine de Fourier, et ne sont pas adaptés pour la tomographie locale.

L'approche que nous proposons, pour répondre au problème de la tomographie locale, est différente des approches précédentes car elle s'appuie sur les travaux de M. Holschneider [19] pour inverser la transformée de Radon : ici la transformée de Radon d'une fonction est vue comme une transformée en ondelettes singulière, et la fonction peut être directement reconstruite à partir de ses projections, simplement par une inversion de la transformée en ondelettes 2D continue. Dans une étude plus poussée [9], nous montrons qu'en fait l'approche de M. Holschneider peut être ramenée à une décomposition en ridgelets biorthogonales, mais bien avant l'heure (1991!). Encore une fois l'originalité de cette approche réside dans l'utilisation d'ondelettes (en fait ridgelets) distributions : sur le plan théorique, on perd la stabilité $L^{2}$ de la transformée (qui est le principe de l'approche par ondelettes/vaguelettes), mais sur le plan pratique, on gagne une étape par rapport aux méthodes précédentes : celle du calcul des coefficients d'ondelettes (ou de vaguelettes) de la transformée de Radon (ce qui divise les calculs par 2). Nous montrons dans les tests numériques que pour le problème qui nous intéresse, celui de la reconstruction globale ou partielle, à partir de données complètes ou tronquées, de fonctions constantes par morceaux, la méthode de M. Holschneider est néanmoins robuste, et s'adapte parfaitement au contexte local.

Bien que la transformée de Radon, comme la transformée en ondelettes, soient définies pour des fonctions $f$ de $L^{2}(\mathbb{R})$, nous considèrerons dans la suite, pour simplifier, que les fonctions $f$ étudiées sont dans $\mathcal{S}\left(\mathbb{R}^{2}\right)$ (espace des fonctions $C^{\infty}$ à décroissance rapide ainsi que leurs dérivées). Dans un premier temps nous étendons la définition de la transformée en ondelettes (1) à des ondelettes d'analyse g, qui sont des distributions tempérées :

$$
W_{g} f(a, b, \alpha)=<T_{b} D_{a} R_{\alpha} g, f>
$$

où les opérateurs $T_{b}, D_{a}$ et $R_{\alpha}$ sont définis dans l'espace des distributions $\mathcal{S}^{\prime}\left(\mathbb{R}^{2}\right)$ par $: \forall \varphi \in \mathcal{S}\left(\mathbb{R}^{2}\right)$,

$$
<T_{b} g, \varphi>=<g, \varphi(x+b)>, \quad<D_{a} g, \varphi>=<g, a \varphi(a x)>, \quad<R_{\alpha} g, \varphi>=<g, \varphi\left(r_{\alpha} x\right)>
$$

L'idée originale de M. Holschneider [19] est de considérer comme "ondelette" (avec un abus de langage, car une ondelette par définition est une fonction auto-admissible) la distribution suivante :

$$
g=\delta\left(x_{1}\right) 1\left(x_{2}\right): f \in \mathcal{S}\left(\mathbb{R}^{2}\right) \mapsto \int_{\mathbb{R}} f\left(0, x_{2}\right) d x_{2}
$$


Le coefficient d'ondelette est ainsi directement relié à la transformée de Radon (8) par :

$$
<T_{b} D_{a} R_{\theta} g, f>=\mathcal{R}_{\theta} f\left(\mathbf{b} . \mathbf{u}_{\theta}\right)
$$

Pour inverser la transformée de Radon il suffit alors d'inverser cette transformée en "ondelettes" en utilisant une ondelette de synthèse admissible $h$ :

$$
f(\mathbf{x})=\frac{1}{C_{g h}} \int_{0}^{+\infty} \iint_{\mathbb{R}^{2}} \int_{0}^{2 \pi} \mathcal{R}_{\theta} f\left(\mathbf{b} . \mathbf{u}_{\theta}\right) \frac{1}{a} h\left(r_{-\theta}\left(\frac{\mathbf{x}-\mathbf{b}}{a}\right)\right) \frac{d a}{a^{3}} \mathbf{d} \mathbf{b} d \theta
$$

et dans ce cas la condition d'admissibilité s'écrit :

$$
C_{g h}=\int_{\mathbb{R}} \frac{\hat{h}\left(k_{1}, 0\right)}{\left|k_{1}\right|^{2}} d k_{1}<+\infty
$$

ce qui signifie que $h$ a 2 moments nuls dans la direction $x_{1}$ :

$$
\iint h\left(x_{1}, x_{2}\right) d x_{1}=\iint x_{1} h\left(x_{1}, x_{2}\right) d x_{1}=0
$$

On peut donc utiliser dans les calculs de reconstruction une ondelette $h$ classique, c'est à dire une fonction à décroissance rapide, et possédant deux moments nuls, comme par exemple le Laplacien de la Gaussienne, $h=\Delta G$. Contrairement à la formule de rétroprojection filtrée (9), cette formule d'inversion intègre la transformée de Radon contre des fonctions locales.

En pratique on utilisera plutôt la transformée en ondelettes dyadique avec une ondelette de synthèse $\tilde{h}$ radiale pour inverser la transformée de Radon :

$$
f(\mathbf{x})=\sum_{j=-\infty}^{+\infty} 4^{-j} \iint_{\mathbb{R}^{2}}\left[\int_{0}^{2 \pi} \mathcal{R}_{\theta} f\left(\mathbf{b} \cdot \mathbf{u}_{\theta}\right) d \theta\right] \quad \frac{1}{2^{j}} \tilde{h}\left(2^{-j}(\mathbf{x}-\mathbf{b})\right) \mathbf{d b}
$$

où par exemple $\tilde{h}$ a pour transformée de Fourier en $\mathbf{k}=\left(k_{1}, k_{2}\right)$ (où $h=\Delta G$ ) :

$$
\widehat{\tilde{h}}(\mathbf{k})=\frac{\hat{h}(\mathbf{k})}{\sum_{j=-\infty}^{+\infty} \hat{h}\left(2^{j} k_{1}, 0\right)}=\frac{\|\mathbf{k}\|^{2} e^{-\pi\|\mathbf{k}\|^{2}}}{\sum_{j=-\infty}^{+\infty} 4^{j} k_{1}^{2} e^{-\pi 4^{j} k_{1}^{2}}}
$$

La formule (10) est bien adaptée au problème intérieur : pour reconstruire $f(\mathbf{x})$ en un point $\mathbf{x}$ appartenant à la région d'intérêt, il suffit de restreindre dans la formule (10) (ou (11) pour le cas discret), le domaine d'intégration sur les échelles $a$, correspondant au rayon de la région d'exposition.

Dans [11], nous détaillons le calcul pratique de la reconstruction locale, et nous présenterons dans [9] de nombreux tests de reconstruction, dont certains sont reproduits au paragraphe suivant. De plus, notre méthode est extensible à des données Radon sur un section angulaire $\left(\theta \in\left[\theta_{1}, \theta_{2}\right]\right)$, en modifiant l'intégrale sur les angles dans (10), et en utilisant une ondelette de reconstruction directionnelle [9].

\subsection{Tests numériques}

Nous avons testé la méthode de reconstruction locale sur le problème classique du fantôme de Shepp Logan (figure 3(a), gauche). On compare tout d'abord la reconstruction obtenue avec l'algorithme classique de Rétro-Projection Filtrée (FBP) et celle fournie par notre méthode, pour des données globales. Les résultats sont présentés à la figure 3(a), et qualitativement les images reconstruites se ressemblent. Les coupes à travers trois sections différentes sont très proches, avec toutefois un phénomène de Gibbs plus important pour la reconstruction FBP (figure 3(b)).

Dans le cas de données locales correspondant au problème intérieur, la région d'intérêt est définie sur la figure 4, ainsi que la région d'exposition, légèrement plus grande. Les données Radon sont issues uniquement de la région d'exposition, qui correspond en réalité à la zone de radiations administrées au patient.

Nous présentons deux exemples de reconstruction dans la région d'intérêt, à partir de ces données : le premier concerne une région d'intérêt au centre du fantôme (ROI : $16 \times 16$ pixels; ROE : $28 \times 28$ pixels); le deuxième concerne une région d'intérêt décentrée (figure 5). Dans les deux cas, les reconstructions obtenues sont satisfaisantes, car elles permettent de bien localiser les discontinuités du fantôme. Dans le cas d'une région d'intérêt centrée, nous pouvons donner quelques éléments de comparaison avec la méthode de [27] : tout d'abord sur le plan visuel, les résultats de reconstruction sont similaires, pour un taux d'exposition identique (environ $22 \%$ ). Cette comparaison pourrait être affinée en visualisant des coupes 


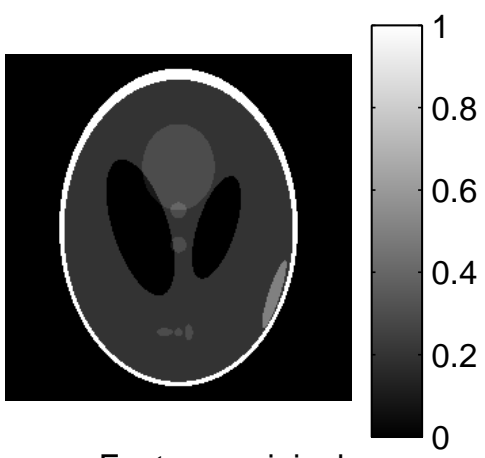

Fantome original

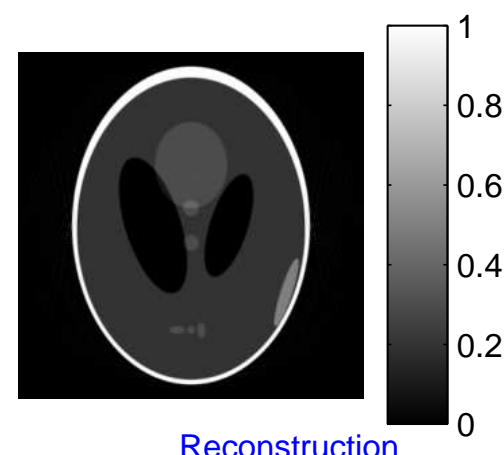

Reconstruction avec la methode FBP

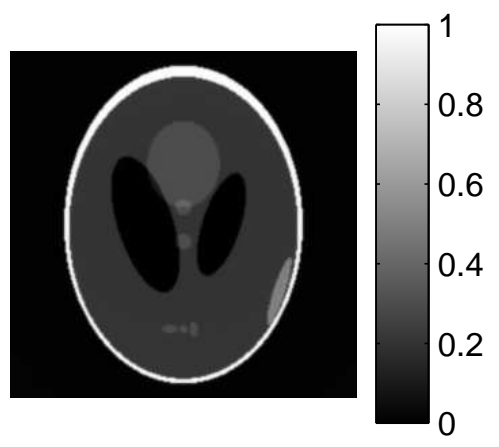

Ondelettes

(a) Reconstructions globales

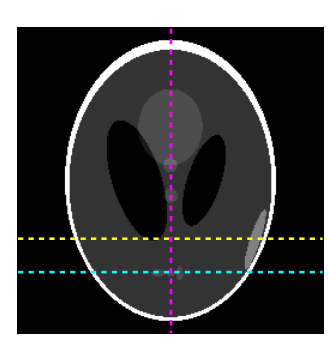

(b) Coupes

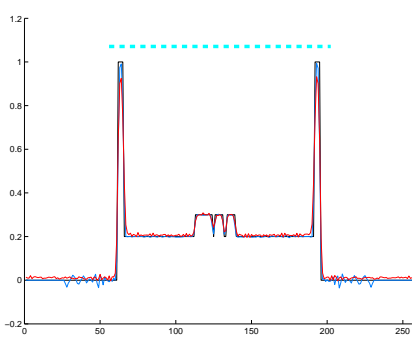

(c) Coupe 1 (en noir : fantôme; en bleu : FBP; en rouge : ondelettes)

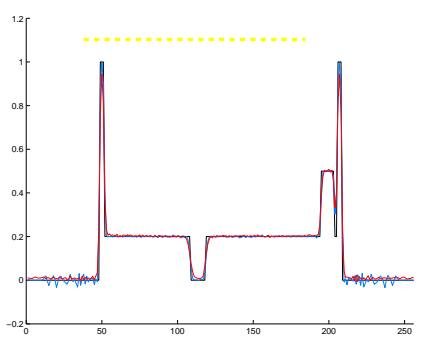

(d) Coupe 2

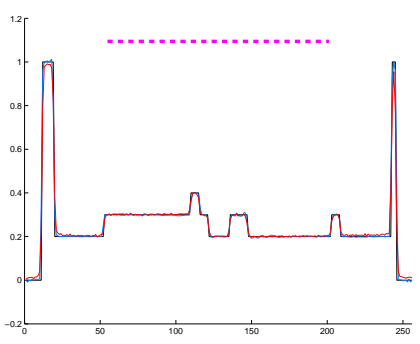

(e) Coupe 3

FiguRE 3. Inversion de la Transformée de Radon en utilisant des données complètes.

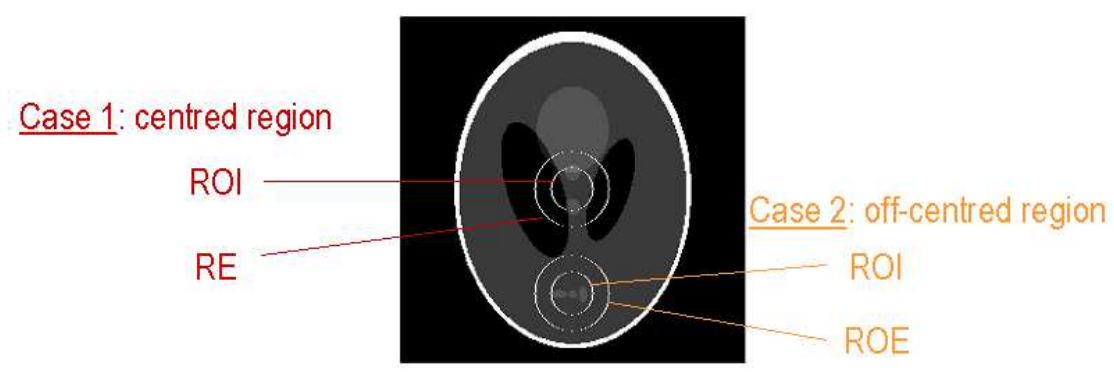

FigurE 4. Définition des régions d'intérêt, et des régions d'exposition associées

transversales, qui malheureusement ne sont pas disponibles dans [27]. Les complexités algorithmiques des reconstructions, pour les deux méthodes sont du même ordre de grandeur que la FBP $\left(O\left(N^{3}\right)\right.$ pour une image de taille $N^{2}$ ) : mais dans notre méthode, on gagne une étape de calcul en assimilant directement les coefficients de Radon à des coefficients d'ondelettes (tandis que dans [27], la méthode inclut une transformée en ondelette unidimensionnelle de la transformée de Radon, pour tous les angles).

Nous nous intéressons dans un dernier exemple au problème à angle limité : si les approches de reconstruction par ondelettes 2D tensorielles (approches de Berenstein-Walnut [8], Donoho [15]) sont a priori bien adaptées au problème intérieur, elles deviennent non praticables pour des problèmes à angle limité : il s'agit dans ce cas de reconstruire la fonction $f$ dans un secteur angulaire du plan, à partir de la connaissance de sa transformée de Radon $\mathcal{R}_{\theta} f$ pour des angles $\theta$ correspondant à ce secteur. Cette situation se produit par exemple quand les bords d'un lit empêchent l'acquisition des données scanner tout atour d'un patient. Dans ces conditions, seules les transformées présentant un paramètre angulaire sont adaptées, comme les décompositions en ridgelets, curvelets, et la méthode basée sur la transformée en ondelettes continue directionnelle. Le fantôme de la figure 6 est proposé par Natterer dans [5] (chap V.2). Nous comparons notre méthode (figure 6 droite) à la méthode de rétroprojection filtrée sur les données tronquées prolongées par continuité (figure 6 centre). On constate que sur ces données tronquées, la méthode par ondelettes fournit, comme la rétroprojection filtrée, une reconstruction satisfaisante des discontinuités verticales, tout en présentant des artefacts moindres au coeur de la région d'intérêt. 

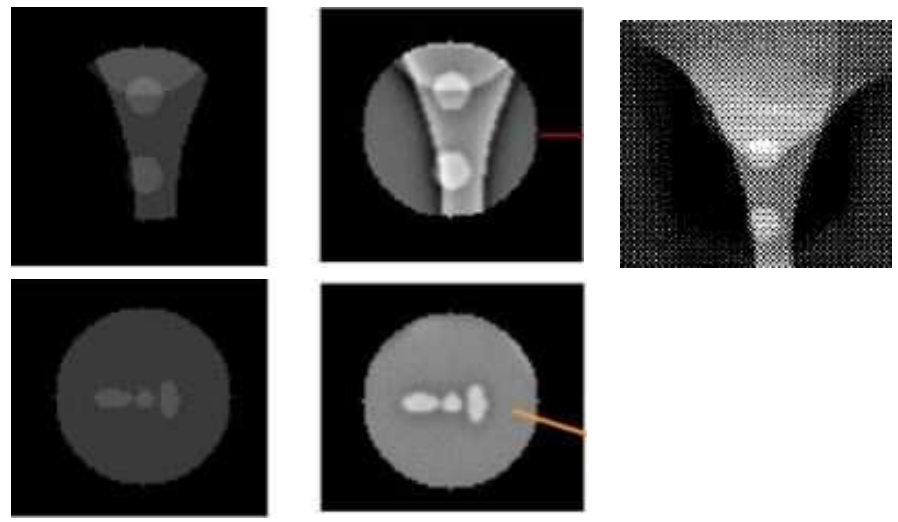

Figure 5. Reconstructions locales. Première ligne : zoom sur la région d'intérêt centrée, reconstruction par ondelettes dans la région d'intérêt et résultat extrait de [27]; Deuxième ligne: zoom sur la région d'intérêt décentrée, et reconstruction locale par ondelettes
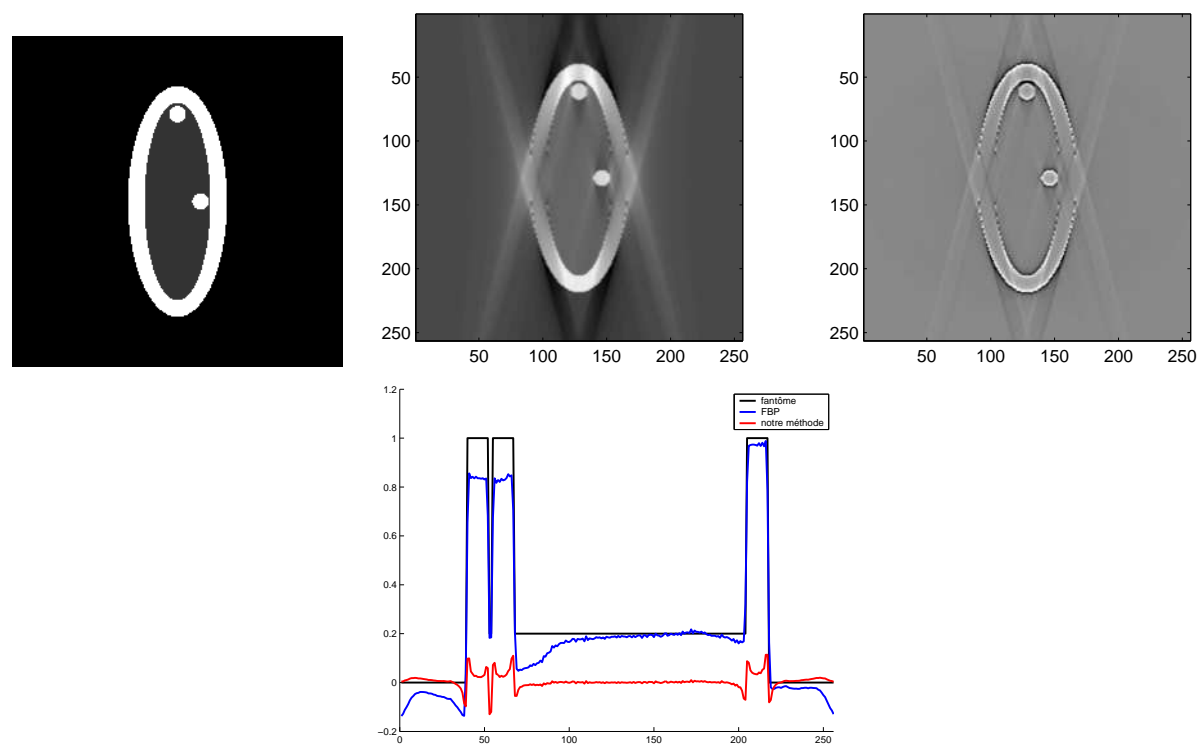

FIGURE 6. Reconstructions dans le cas du problème à angle limité; seules les mesures effectuées pour $\theta \in[15,165]$ sont conservées. Première ligne : le fantôme et les reconstrutions obtenues par rétroprojection filtrée (milieu) et par ondelettes (à droite). En deuxième ligne, sections verticales : en noir du fantôme, en bleu de la rétroprojection filtrée, en rouge de la méthode par ondelettes.

\section{Segmentation D'images par ondelettes en Vue de la ReConstruction 3D D'UNE SURFACE DE VERTÈBRE}

Le scanner est une solution efficace pour la reconstruction d'objets 3D mais invasive et souvent non praticable en cours d'intervention (à cause de l'encombrement de l'appareil et de l'intensité des radiations). Or dans de nombreuses applications, l'utilisation de techniques d'imagerie interventionnelle peut apporter l'avantage, parfois crucial, d'une information sur le patient tel qu'il est lors de l'intervention et non tel qu'il était dans les conditions de l'imagerie pré-opératoire. Pour réduire le caractère invasif de l'imagerie, d'autres solutions que le scanner sont alors envisagées.

\subsection{Reconstruction 3D d'une surface de vertèbre en chirurgie orthopédique}

Dans cette étude, nous nous sommes intéressés à la mise en place d'un module d'assistance par ordinateur en chirurgie orthopédique, dans le cas du vissage pédiculaire (vissage d'une vis dans le pédicule d'une vertèbre). L'objectif ici est de guider le chirurgien au cours de l'intervention, en lui proposant une navigation en temps-réel de ses instruments dans une vue tridimensionnelle de la vertèbre. L'intervention est supposée se dérouler avec fluoroscopie, c'est à dire que l'on dispose au début de l'opération, de seulement deux radiographies de la vertèbre prises de face et de profil. L'objectif consiste alors, à partir de ces deux radiographies, à reconstruire la surface de la vertèbre en trois dimensions. 
La méthodologie mise au point par Desbat, Fleute et Lavallée dans $[16,17]$ pour reconstruire la surface 3D de la vertèbre consiste à utiliser un modèle statistique de forme, de la surface d'une vertèbre. A partir d'une base de données de surfaces de vertèbres (schématisée à la figure 7), on déduit une forme moyenne pour la surface et des déformations admissibles (par décomposition en composantes principales). La surface de la vertèbre observée est alors cherchée parmi les formes admissibles données par le modèle, à partir des contours (pas forcément extérieurs) de la vertèbre sur les deux radiographies. Cette recherche de surface s'effectue par un algorithme itératif de recalages élastiques du modèle statistique de formes $3 \mathrm{D}$ à partir de contours 2D. Jusqu'alors, les tests de reconstruction ont été effectués par des contours initiaux déterminés à la main. L'objectif consiste maintenant à définir et implémenter en temps-réel une méthode automatique de détection de contours, adaptée aux images radiographiques.
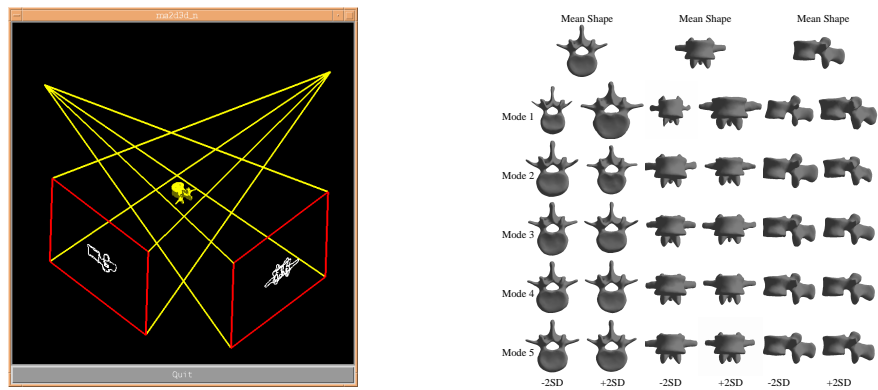

FIGURE 7. Reconstruction d'une surface 3D de vertèbre à partir de 2 radiographies segmentées (à gauche), et d'une base de données de vertèbre à laquelle on associe une forme de surface moyenne et des modes de déformation (à droite) [16].

\subsection{Détection automatique de contours par ondelette vectorielle}

La détection des contours d'un objet dans une image est un problème fondamental de traitement d'image. Dans la mesure où nous nous sommes intéressés à la fois à des contours extérieurs, et à des contours intérieurs de la vertèbre (figure 8 en haut à droite), nous utilisons une approche par "gradient", avec un détecteur de bord multi-échelles.

- Dans un premier temps, les contours, qui correspondent aux brusques variations de l'intensité, sont définis comme des courbes de singularité de l'image, le long desquelles l'image est régulière. En pratique, ces courbes sont caractérisées par des points qui sont des maxima locaux du gradient de l'intensité le long des lignes de courant du gradient. Chacune de des lignes de contour suit une direction orthogonale au gradient de l'intensité, et est caractérisée par une régularité Lipschitzienne variant peu. Ce paramètre de régularité va permettre de classifier les différentes courbes obtenues.

- Pour détecter les contours ainsi définis, nous utilisons une transformée en ondelettes bidimensionnelle, avec comme ondelette d'analyse un gradient de Gaussienne (exemple 2 de la section 2), associée à la technique de suivi des lignes de maxima des coefficients d'ondelettes définie par Mallat-Hwang [23]. Dans le cas de la dimension 2 d'espace, cette méthode est généralisée à une ondelette vectorielle, donc directionnelle, et nous avons défini et implémenté un algorithme rapide de calcul des lignes de maxima, utilisant des filtres discrets en cascade [20].

Les principales étapes de l'algorithme de segmentation sont les suivantes :

(1) Calcul de la transformée en ondelettes à différentes échelles a de l'intensité de la radiographie $\mathbf{W}_{\nabla G}(a, \mathbf{b})$, suivant la formule (5).

(2) A chaque échelle $a$, recherche des maxima locaux de $\left\|\mathbf{W}_{\nabla G}(a, \mathbf{b})\right\|$ dans la direction du vecteur $\mathbf{W}_{\nabla G}(a, \mathbf{b})$.

(3) Calcul des lignes de maxima par chanage de ces maxima locaux à travers les échelles. La localisation d'un maximum local de l'échelle la plus fine (en pratique 1), qui appartient à une ligne de maxima suffisament longue fournit la localisation d'un point de contour.

(4) Estimation de la régularité Lipschitzienne de l'intensité en tout point de contour, par analyse du comportement à travers les échelles de la ligne de maxima correspondante [23]. Ce paramètre permet d'éliminer facilement certains points de contours n'appartenant pas à la vertèbre.

(5) Fabrication de lignes de contours à partir des points de contours (isolés) précédents, en reliant les points dans les directions orthogonales à $\mathbf{W}_{\nabla G}(1, \mathbf{b})$ (1 désignant l'échelle la plus fine).

La méthode de détection et de classification des contours ainsi définie a été testée pour des images de référence, et qui correspondent à des contours "test" (marches, lignes ou points isolés). A la figure 8 nous présentons les points de contours détectés, avec leur régularité locale associée (en couleur) sur trois images : une image géométrique sur laquelle les 3 types de contours sont présents, une image constituée 

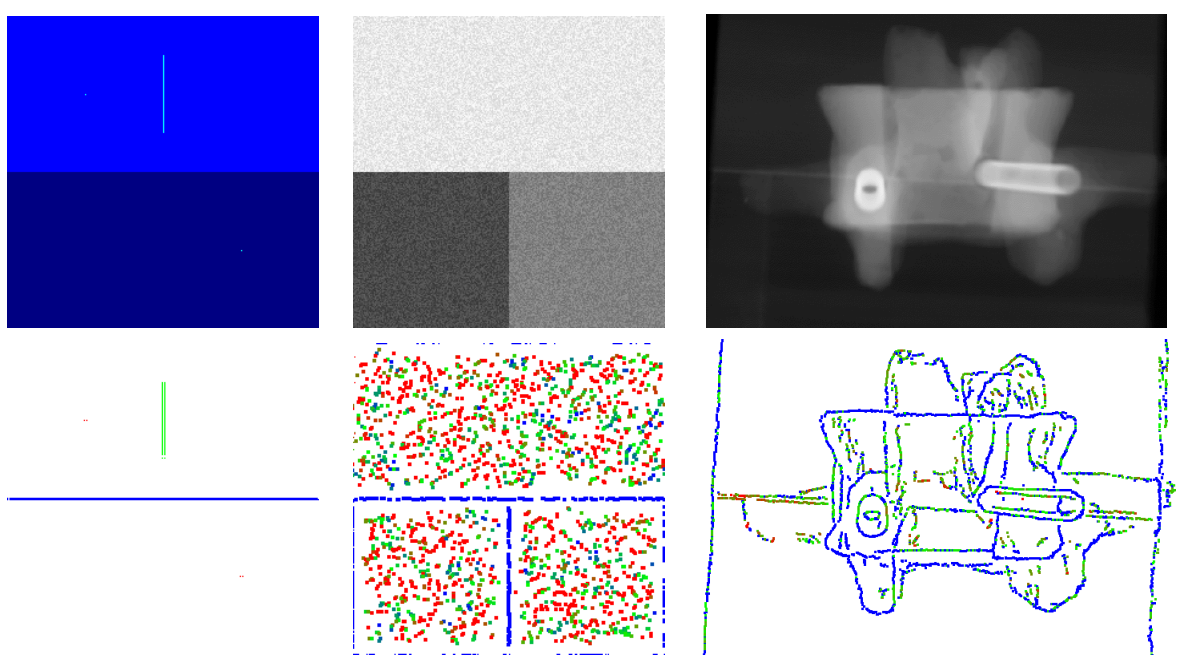

FIGURE 8. Détection de points de contours par suivi des lignes de maxima jusqu'à l'échelle la plus fine. La couleur représente le paramètre de régularité locale associé à chaque point de contours.

de marches bruitée, et une radiographie de vertèbre isolée. Pour les deux premières images, les résultats sont proches de la théorie, les marches étant détectées par des points de régularité proche de 0 , les lignes par des points de régularité proche de -1, et les points isolés, ainsi que le "bruit", correspondent à des points de contours de "régularité" voisine de -2 (diracs bidimensionnels). Dans la troisième image, le bruit peut alors facilement être distingué des contours de la vertèbre qui ont une régularité voisine de 0 (correspondant à une discontinuité de l'intensité) .

\subsection{Reconstruction $3 \mathrm{D}$ et comparaison avec une méthode de contours actifs}

Pour évaluer quantitativement la précision de l'algorithme complet de reconstruction d'une surface de vertèbre, nous avons effectué une série de tests sur des données de synthèse [10] : à partir d'une surface de vertèbre définie par un maillage, et donc parfaitement connue, nous avons simulé des radiographies de cette vertèbre d'une part sans environnement, et d'autre part, en plongeant chacune des coupes dans un scanner réel, dans un environnement réaliste (figure 9, ligne 1). Nous avons ensuite effectué des tests de reconstruction, pour lesquels nous avons pu évaluer précisément l'erreur par rapport à la surface de référence. De manière plus détaillée, nous avons dans un premier temps segmenté les radiographies par l'algorithme précédent (ligne 2), puis sélectionné les lignes de contours significatifs (ligne 3) qui vont servir d'initialisation à l'algorithme de reconstruction 3D, basé sur le modèle statistique déformable. La dernière ligne de la figure 9 représente les sections horizontales et verticales des surfaces reconstruites, superposées aux sections réelles de la vertèbre étudiée. Les résultats de reconstruction obtenus sont satisfaisants du point de vue clinique (moins de 4 pixels d'écart, soit $2 \mathrm{~mm}$ au niveau des pédicules).

Nous avons ensuite comparé dans [10] les résultats de cette méthode avec une méthode de contours actifs GVF (Gradient Vector Flow [28]), qui nécessite également une intervention de l'utilisateur, mais ici pour définir le contour initial. Cette deuxième méthode, sur les radiographies que nous considérons, va permettre de détecter le contour extérieur de la vertèbre, et présente l'avantage de définir un contour fermé, mais le désavantage de ne pas détecter les parties de contours intérieurs (voir figure 10).

Nous avons ensuite comparé dans les deux cas les résultats de reconstruction de vertèbre 3D, en appliquant l'algorithme du modèle statistique sur les points détectés, et pour cette étude, la segmentation par ondelettes des radiographies conduit à une meilleure reconstruction 3D, en particulier parce que les contours intérieurs sont importants et ne sont pas détectés par la méthode de contours actifs GVF [10]. Une amélioration de la méthode de segmentation consisterait à un mélange du détecteur par ondelettes et de la méthode GVF : toutefois, dans le cas de vertèbres isolées, les résultats actuels sont satisfaisants, car les erreurs commises sont du même ordre de grandeur que celles de l'algorithme de reconstruction. Cette position sera à reconsidérer en conditions réelles d'opérations, quand de nombreuses parties de la vertèbre ne sont pas visibles sur la radiographie.

\section{RÉFÉRENCES}



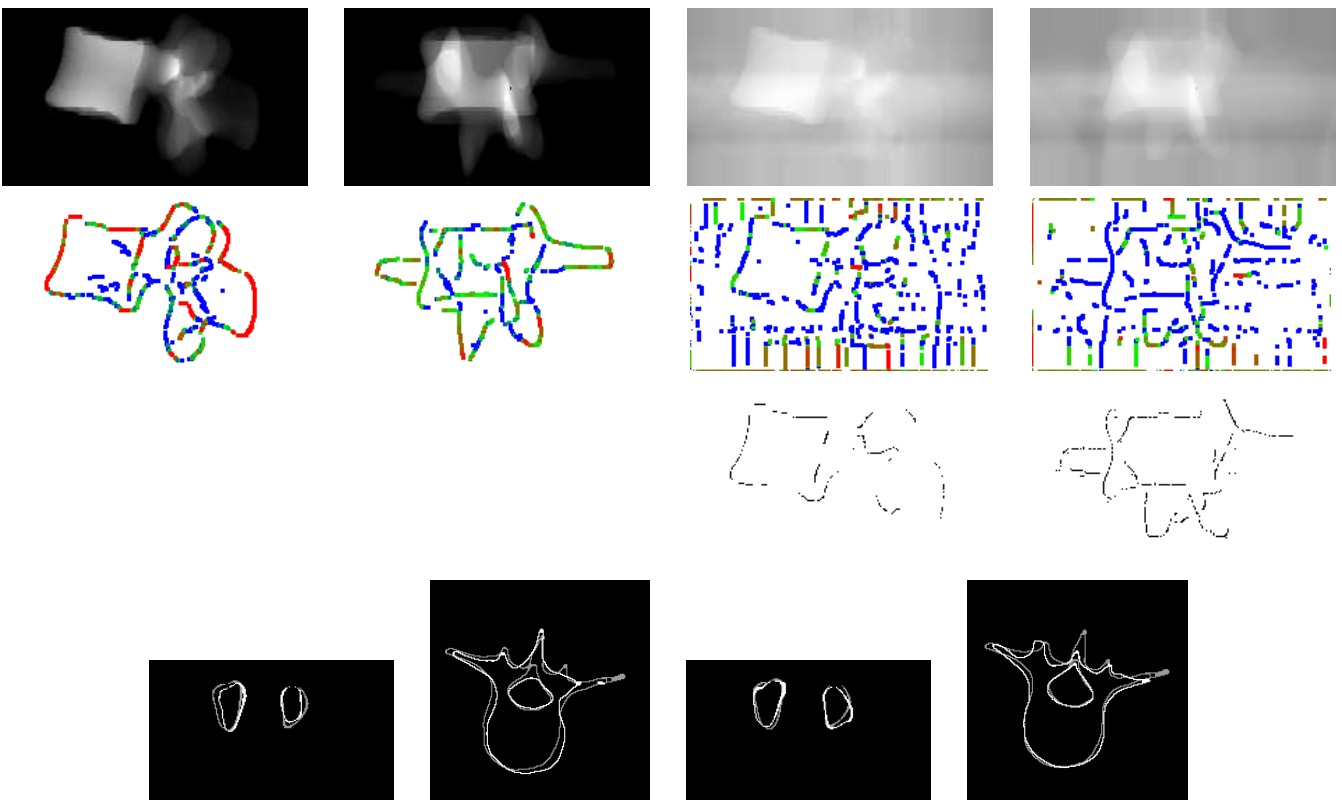

FIGURE 9. (Ligne 1) Radiographies simulées d'une vertèbre isolée (2 premières figures) et d'une vertèbre plongée dans un vrai scanner. (Ligne 2) segmentation des radiographies par l'algorithme. (Ligne 3) Extraction par l'expert de contours significatifs. (Ligne 4) sections horizontales et verticales des surfaces reconstruites (la vertèbre de référence est en gris, la vertèbre reconstruite est en blanc).

\begin{tabular}{|c|c|}
\hline $\begin{array}{c}\text { Complete contours } \\
\text { (reference) }\end{array}$ & $\begin{array}{c}\text { Phantom of dry } \\
\text { vertebra } \\
-\end{array}$ \\
GVF method & \\
\hline $\begin{array}{c}\text { Phantom of dry } \\
\text { vertebra } \\
-\end{array}$ \\
Wavelet method \\
\hline $\begin{array}{c}\text { Phantom of real } \\
\text { vertebra } \\
-\end{array}$ \\
GVF method
\end{tabular}

FigurE 10. Détection de contours avec les deux méthodes (ondelettes ou GVF), et comparaison avec les contours de référence.

[2] Special issue on "Wavelets in Medical Imaging", M. Unser, A. Aldroubi and A. Laine eds, IEEE Transactions on Medical Imaging, 22(3), 2003.

[3] S. Mallat, Une exploration des signaux en ondelettes, Les Editions de l'Ecole Polytechnique, 2000.

[4] J.P. Antoine, R. Murenzi, P. Vandergheynst, and S. Twareque Ali, Two-dimensional wavelets and their relatives, Cambridge university press, 2004.

[5] F. NATTERER, The mathematics of computerized tomography, Wiley, 1986.

[6] B. TorrésAni, Analyse continue par ondelettes, CNRS Editions, Paris, 1995. 
[7] B. Alfano, U. Amato, A. Antoniadis, M. Larobina. Segmentation of magnetic resonance brain images through discriminant analysis, Journal of Neuroscience Methods, Volume 131, Issues 1-2, 65-74, 2003.

[8] C. Berenstein, D. Walnut, Local inversion of the radon transform in even dimensions using wavelets, In 75 years of Radon Transform, S. Gindikin and P. Michor eds, 45-69. International Press, Cambridge, MA, 1994.

[9] A. Bilgot, Méthodes locales d'identification de surfaces de discontinuité à partir de projections tronquées pour l'imagerie interventionnelle, Thèse de l'université Joseph Fourier, Grenoble I, en préparation.

[10] A.Bilgot, O. Le Cadet, V. Perrier, L. Desbat, Edge detection and classification in X-Ray images. Application to interventional 3D vertebra-shape reconstruction, SURGETICA 2005 : Gestes médico-chirurgicaux assistés par ordinateurs : outils et applications, 459-467 Sauramps Medical 2005.

[11] A.Bilgot, V. Perrier, L. Desbat, Wavelets, Local Tomography and Interventional X-Ray Imaging, IEEE NSS/MIC2004 (Nuclear Science Symposium/Medical Image Conference), Rome, 2004.

[12] J. CAnny, A computational approach to edge detection, IEEE Trans. Patt. Anal. and Mach. Intell., 36, 961-1005, 1986.

[13] E.J. Candìs, D.L. Donoho, Recovering edges in ill-posed inverse problems. Optimality of curvelets frames, Ann. Statist. 30, 784-842 (2000).

[14] E.J. CAndìs, F. GuO, New multiscale transforms, minimum total variation synthesis : Applications to edgepreserving image reconstruction, Signal Processing, 82(11), 1519-1543, 2002.

[15] D.L. Donoho, Nonlinear solution of linear inverse problems by wavelet/vaguelette decomposition, Appl. Comput. Harm. Anal. 2 : 101-126, 1995.

[16] M. Fleute, Shape reconstruction for computer assisted surgery based on non-rigid registration of statistical models with intra-operative point data and X-ray images, Thèse de l'Université Joseph Fourier, Grenoble I, 2001.

[17] M. Fleute, S. Lavallée And L.Desbat, Integrated Approach for Matching Statistical Shape Models with Intra-operative 2D and 3D Data. In T. Dohi and R. Kikinis editors, MICCAI 2002, LNCS 2489, pp 364-372, 2002. Springer-Verlag, 2002.

[18] W. T. Freeman, E. H. Adelson, The Design and Use of Steerable Filters, IEEE Trans. Patt. Anal. and Mach. Int. 13(9), 891-906, 1991.

[19] M. Holschneider, Inverse Radon Transform through inverse wavelet transform, Inverse problems, 7, 853861, 1991.

[20] O. Le CADET, Détection et caractérisation des contours d'une image. Application à la segmentation d'images médicales et au watermarking, PhD thesis INP Grenoble (2004).

[21] N. LEE, B. LucIER, Wavelet methods for inverting the Radon Transform with noisy data, IEEE Trans. Image Processing, 10, 2001.

[22] E. LePennec And S. Mallat, Sparse Geometric Image Representation with Bandelets, IEEE Trans. on Image Processing 14(4), 423-438, 2005.

[23] S. Mallat and W.L.H. Hwang, Singularity detection and processing with wavelets, IEEE Trans. Info. Theory, 38(2), 617-643, 1992.

[24] S. Mallat And S. Zhong, Characterization of signals from multiscale edges, IEEE Trans. Patt. Anal. and Mach. Intell., 14(7), 710-732, 1992.

[25] R. Murenzi, Transformée en ondelettes multidimensionnelle et application à l'analyse d'images, Thèse Louvain-La-Neuve, 1989.

[26] G. Peyré, Géométrie multi-échelles pour les images et les textures, Thèse de l'Ecole Polytechnique, 2005.

[27] F. Rashid-Farrokhi, K.J. R. Liu, C.A. Berenstein, D.F. Walnut, Wavelet-based multiresolution local tomography, IEEE Trans. Image Processing 6(10), 1412-1430, 1997.

[28] C. Xu, J.L. Prince, Snakes, shapes and Gradient Vector Flow, IEEE Trans. Image Processing 7(11), 1998. 